\title{
Korovkin-type approximation by operators in Riesz spaces via power series method
}

https://doi.org/10.1515/dema-2019-0041

Received June 13, 2019; accepted October 3, 2019

Abstract: In this paper we prove an Ozguç, Yurdakadim and Taş version of the Korovkin-type approximation by operators in the sense of the power series method. That is, we try to extend the Korovkin approximation theorems, obtained by Ozguç and Taş in 2016, and Taş and Yurdakadim in 2017, for concrete classes of Banach spaces to the class of Riesz spaces. Some applications are presented.

Keywords: Riesz spaces, uniformly convergence, power series method

MSC 2010: 06F25, 46A40

\section{Introduction and preliminaries}

Approximation theory has many important applications in polynomial theory, functional analysis, numerical solutions of differential and integral equations. Also, it finds its motivation in many mathematical problems coming from other areas such as industrial applications or economic modeling. Perhaps the most striking results in the classical approximation theory are the Weierstrass and Korovkin-type Approximation theorems which play an important role in approximation theory. In particular, in 1953 Korovkin proved a very useful and simple criterion for whether a given sequence of positive linear operators in $C([a, b])$, the space of all continuous real-valued functions defined on $[a, b]$, is an approximation process by providing the convergence only on the three test functions $\left\{1, x, x^{2}\right\}$ (see [1]). Using various types of convergence with a variety of assumptions on the processes, many mathematicians have worked on generalizing the Korovkin theorems in several ways. First, we recall [2] and [3] where some approximation theorems by means of positive linear operators acting on more general functions spaces are obtained. In [4], Duman and Orhan studied the concept of $\mathcal{A}$-statistical convergence on $C(X, \mathbb{R})$, the space of all real-valued continuous functions on a compact Hausdorff space $X$. A few years later in [5], Guessab and Schmeisser generalized the classical Korovkin theorem for positive linear operators on $C(\Omega)$ spaces. Later in [6], Atlihan and Taş proved a Korovkin-type Approximation theorem with respect to $\mathcal{A}$-summation processes. A Banach lattice version of Korovkin's theorem has been established and is due to Wiśniewska and Wójtowicz (see [7]). Very recently, in [8] the first author, Dorai and Wójtowicz gave a Riesz space version of the Korovkin theorem. Korovkin-type Approximation theorems have been greatly improved using more general convergences such as statistical, filter convergences and convergences generated by the class of summability methods via power series methods which includes both Abel and Borel methods (see e.g., [9-14]). However, in spite of the progress made in the development of the Korovkin-type Approximation theory via power series methods, in the general case of Banach lattices or Riesz spaces it has received little or no attention. The main purpose of this paper is to take a step in this direction. In fact, this paper is mainly concerned with an abstract version of the Korovkin-type Approximation theorems for a sequence of

\footnotetext{
^Corresponding Author: Elmiloud Chil: University of Tunis, Institut préparatoire aux études d'ingenieurs de Tunis, 2 Rue jawaher lel Nehrou Monflery 1008, Tunisia; E-mail: Elmiloud.chil@ipeit.rnu.tn Marwa Assili: L.A.T.A.O. Faculty of sciences of Tunis University, ELManar Compus universitaire Elmanar, Tunisia; E-mail: marwa.assili23@gmail.com
} 
operators acting on Riesz spaces using summability methods via power series methods. The present paper is largely motivated by the works $[10,13]$. Actually, Banach lattices and positive operators in $[10,13]$ will be replaced by Riesz spaces and order bounded disjointness preserving operators respectively. As far as we know, in the literature there are no works fully dedicated to this kind of problems in the framework of Riesz spaces. For a detailed account of the Korovkin-type Approximation theory we refer the readers to [3].

For the terminology, notation and concepts related to Riesz spaces (i.e., vector lattices) not explained or proved in this paper the reader is encouraged to consult the standard monographs [14].

All (real) Riesz spaces under consideration are assumed to be Archimedean and the only topology we consider in this paper is the (relatively) uniform topology (cf. [15, Sections 16 and 63]).

A linear mapping $T$ defined on a Riesz space $E$ with values in a Riesz space $F$ is called order bounded (in symbols, $T \in \mathcal{L}_{b}(E, F)$ ) if $T$ maps order intervals into order intervals. An operator $T$ is called a Riesz (or lattice) homomorphism if $T(x) \wedge T(y)=T(x \wedge y)$ (equivalently, $T(x) \vee T(y)=T(x \vee y)$ ) for all $x, y \in E$. An operator $T$ is a Riesz homomorphism if and only if $T(|x|)=|T(x)|$ for all $x \in E$. An operator $T$ is called disjointness preserving if, for all $x, y \in E,|T(x)| \wedge|T(y)|=0$ whenever $|x| \wedge|y|=0$. More details about Riesz homomorphisms and order bounded disjointness preserving operators can be found in [14].

The following paragraph deals with power series. A series in an Archimedean Riesz space $E$ is an infinite sum $\sum_{n=0}^{+\infty} a_{n}$, where $a_{n} \in E$ for all $n \in \mathbb{N}$. We say that $\sum_{n=0}^{+\infty} a_{n}$ converges uniformly if the sequence $\left(\sum_{n=0}^{m} a_{n}\right)_{m \geq 0}$ of partial sums has relatively uniformly limit in E. Moreover, $\sum_{n=0}^{+\infty} a_{n}$ is said to converge absolutely uniformly if $\sum_{n=0}^{+\infty}\left|a_{n}\right|$ converges uniformly. It is readily verified that if $E$ is relatively uniformly complete then any series in $E$ that converges absolutely uniformly also converges uniformly.

We end this section by giving some examples of classical uniformly complete Riesz spaces. Let $X$ be a compact Hausdorff space and $C(X)$ be the Banach algebra of real continuous functions on $X$; then $C(X)$ is a uniformly complete Riesz space. Moreover, a uniformly complete Riesz space need not be a normed Riesz space: consider the Riesz space of all measurable functions on a measurable space which is $\sigma$-Dedekind complete (so uniformly complete) but not a normed Riesz space.

\section{Main results}

We begin by mentioned further concepts we need in what follows.

Let $\left(a_{n}\right)_{n}$ be a real sequence with $a_{0}>0, a_{n} \geq 0$ for every $n \geq 1$, and such that the corresponding power series $f(t)=\sum_{n=0}^{+\infty} a_{n} t^{n}$ has radius of convergence $0<R \leq+\infty$. Moreover, let $\mathrm{E}$ be a real Riesz space. If, for all $t \in(0, R)$ and a fixed $\left(x_{n}\right)_{n} \in E$,

$$
\sum_{n=0}^{+\infty} a_{n} t^{n} x_{n} \text { converges uniformly and } \lim _{t \rightarrow R^{-}} \frac{1}{f(t)} \sum_{n=0}^{+\infty} a_{n} t^{n} x_{n}=l,
$$

then we say that the sequence $\left(x_{n}\right)_{n}$ is uniformly convergent to $l$ in the sense of the power series method. A well-known class of summability methods are the power series methods, perhaps the most popular ones in this class being Abel's and Borel's method. Indeed, for $R=1, f(t)=\frac{1}{1-t}$ and $a_{n}=1$ for every $n \in \mathbb{N}$, the power series method coincides with Abel method. And, if $R=+\infty, f(t)=e^{t}$ and $a_{n}=\frac{1}{n !}$ for each $n \in \mathbb{N}$, the power series method coincides with the Borel method (see [13]). In order to see that the power series method is more effective than ordinary convergence, let $E=C([0,1])$, the sequence $f_{n}$ in $E$ defined by $f_{2 n}(x)=1$, $f_{2 n+1}(x)=0$ for each $n \in \mathbb{N}$, and $f(t)=e^{t}$. Then $R=+\infty, a_{n}=\frac{1}{n !}$ for each $n \in \mathbb{N}$ and it is easy to see that $\left(f_{n}\right)_{n}$ 
is not uniformly convergent in $E$ but it is uniformly convergent to the constant function $\frac{1}{2}$ in the sense of the power series method because $\lim _{t \rightarrow+\infty} \sup _{x \in[0,1]}\left|\frac{1}{f(t)} \sum_{n=0}^{+\infty} \frac{t^{n}}{n !} f_{n}(x)-\frac{1}{2}\right|=0$ (see [13, p.1294]).

Now we are able to state the main result of this paper, where we prove that $W$, a nonempty subset of $E^{+}$, is a testing set of convergence in the sense of power series for the restrictions of $\left(T_{n}\right)_{n}$, a given sequence of elements of $\mathcal{L}(E, F)$, to $E_{W}$, the ideal generated by $W$.

Theorem 1. Let $E, F$ be two Archimedean Riesz spaces, let $W$ be a nonempty subset of $E^{+}$, and let $\left(T_{n}\right)_{n}$ be a given sequence of elements of $\mathcal{L}(E, F)$ such that:

(a) $\left(T_{n}\right)_{n}$ converges pointwise on $W$ absolutely uniformly in the sense of power series method to $T \in \mathcal{L}(E, F)$.

(b) $T-T_{n}$ is an order bounded disjointness preserving operator.

Then $\left(T_{n}\right)_{n}$ converges pointwise on $E_{W}$ absolutely uniformly in the sense of the power series method to $T$. Thus,

$$
T x=\lim _{t \rightarrow R^{-}} \frac{1}{f(t)} \sum_{n=0}^{+\infty} a_{n} t^{n} T_{n} x
$$

for all $x \in E_{W}$.

Proof. Let us fix $0<y \in W$ and $x \in E_{y}$. Thus, there exists $\lambda>0$ such that $|x| \leq \lambda y$. Since $T-T_{n}$ is an order bounded disjointness preserving operator then $\left|T x-T_{n} x\right| \leq \lambda\left|T y-T_{n} y\right|$, whence

$$
\begin{aligned}
\left|\frac{1}{f(t)} \sum_{n=0}^{+\infty} a_{n} t^{n} T_{n} x-T x\right| & =\left|\frac{1}{f(t)} \sum_{n=0}^{+\infty} a_{n} t^{n}\left(T_{n} x-T x\right)\right| \\
& \leq \frac{1}{f(t)} \sum_{n=0}^{+\infty} a_{n} t^{n}\left|T_{n} x-T x\right| \\
& \leq \frac{\lambda}{f(t)} \sum_{n=0}^{+\infty} a_{n} t^{n}\left|T_{n} y-T y\right| .
\end{aligned}
$$

Now, by the fact that $\left(T_{n}\right)_{n}$ converges pointwise on $W$ absolutely uniformly in the sense of power series to $T$, we have $\lim _{t \rightarrow R^{-}} \frac{1}{f(t)} \sum_{n=0}^{+\infty} a_{n} t^{n}\left|T_{n} y-T y\right|=0$. Therefore,

$$
\lim _{t \rightarrow R^{-}}\left(\frac{1}{f(t)} \sum_{n=0}^{+\infty} a_{n} t^{n} T_{n} x-T x\right)=0
$$

which implies that

$$
T x=\lim _{t \rightarrow R^{-}} \frac{1}{f(t)} \sum_{n=0}^{+\infty} a_{n} t^{n} T_{n} x .
$$

Now let $0 \leq x \in E_{W}$. Thus, $x=x_{1}+. .+x_{k}$, where $x_{i} \in E_{y_{i}}$ for some $y_{1}, \ldots, y_{k}$ in $W$. From the above equalities we have

$$
\lim _{t \rightarrow R^{-}}\left(\frac{1}{f(t)} \sum_{n=0}^{+\infty} a_{n} t^{n} T_{n} x_{i}-T x_{i}\right)=0 \text { and } T x_{i}=\lim _{t \rightarrow R^{-}} \frac{1}{f(t)} \sum_{n=0}^{+\infty} a_{n} t^{n} T_{n} x_{i} \text { for all } 1 \leq i \leq k .
$$

Thus, we have

$$
\lim _{t \rightarrow R^{-}}\left(\frac{1}{f(t)} \sum_{n=0}^{+\infty} a_{n} t^{n} T_{n} x-T x\right)=0 \text { and consequently } T x=\lim _{t \rightarrow R^{-}} \frac{1}{f(t)} \sum_{n=0}^{+\infty} a_{n} t^{n} T_{n} x .
$$

The general case is deduced by linearity since every $x \in E_{W}$ is of the form $x=x^{+}-x^{-}$, where $x^{+}, x^{-} \in E_{W}^{+}$. This completes the proof of the theorem. 
From the preceding theorem it is clear that with respect to the uniformly convergence the singleton $\{x\}$ is a testing set of the uniformly convergence in the sense of the power series method for the restriction of $\left(T_{n}\right)_{n}$ to $E_{\chi}$, the ideal generated by $x$. As an immediate application of this we give the following result.

Corollary 2. Let $X$ be a compact Hausdorff space, $C(X)$ the Riesz space of real continuous functions on $X, F$ an Archimedean Riesz space, $\left(T_{n}\right)_{n}$ a given sequence of elements of $\mathcal{L}(C(X), F)$ and let $T \in \mathcal{L}(C(X), F)$ such that:

(a) $T_{n} 1_{X}$ converges absolutely uniformly in the sense of power series to $T 1_{X}$, where $1_{X}$ is the constant function defined by $1_{X}(x)=1$ for all $x \in X$.

(b) $T-T_{n}$ is an order bounded disjointness preserving operator.

Then $\left(T_{n}\right)_{n}$ converges pointwise on $C(X)$ absolutely uniformly in the sense of the power series method to $T$. Thus,

$$
\operatorname{Tg}=\lim _{t \rightarrow R^{-}} \frac{1}{f(t)} \sum_{n=0}^{+\infty} a_{n} t^{n} T_{n} g
$$

for all $g \in C(X)$.

Note that the preceding theorem gives us a version of Dini's theorem for the power series convergence method as the following corollary shows.

Corollary 3. Let $E, F$ be two Archimedean Riesz spaces, let $W$ be a nonempty subset of $E^{+}$, and let $\left(T_{n}\right)_{n}$ be a given monotone sequence of elements of $\mathcal{L}(E, F)$ such that:

(a) $\left(T_{n}\right)_{n}$ converges pointwise on $W$ absolutely uniformly in the sense of power series method to $T \in \mathcal{L}(E, F)$.

(b) $T-T_{n}$ is a lattice homomorphism for all $n \geq 1$.

Then $\left(T_{n}\right)_{n}$ converges pointwise on $E_{W}$ absolutely uniformly in the sense of power series method to $T$. Thus,

$$
T x=\lim _{t \rightarrow R^{-}} \frac{1}{f(t)} \sum_{n=0}^{+\infty} a_{n} t^{n} T_{n} x,
$$

for all $x \in E_{W}$.

As an immediate application of the preceding corollary we give the following result.

Corollary 4. Let $E, F$ be two Archimedean Riesz spaces, let $W$ be a nonempty subset of $E^{+}$, and let $\left(T_{n}\right)_{n}$ be a given sequence of elements of $\mathcal{L}(E, F)$ such that:

(a) The sequences $\left(T_{n}^{+}\right)_{n}$ and $\left(T_{n}^{-}\right)_{n}$ of positive and negative parts of $\left(T_{n}\right)_{n}$ exist and are monotone.

(b) $\left(T_{n}^{+}\right)_{n}$ and $\left(T_{n}^{-}\right)_{n}$ converge pointwise on $W$ absolutely uniformly in the sense of the power series method respectively to $T^{+}, T^{-} \in \mathcal{L}(E, F)$.

(c) $T^{+}-T_{n}^{+}$and $T^{-}-T_{n}^{-}$are lattice homomorphisms for all $n \geq 1$.

Then $\left(T_{n}\right)_{n}$ converges pointwise on $E_{W}$ absolutely uniformly in the sense of the power series method to $T=T^{+}-T^{-}$. Thus,

$$
T x=\lim _{t \rightarrow R^{-}} \frac{1}{f(t)} \sum_{n=0}^{+\infty} a_{n} t^{n} T_{n} x
$$

for all $x \in E_{W}$.

Before proceeding to our last result, we recall that the power series method is said to be regular if ordinary convergence implies convergence in the sense of the power series method. It is known that the power series method is regular if and only if

$$
\lim _{t \rightarrow R^{-}} \frac{a_{n} t^{n}}{f(t)}=0
$$


(see [16]). Under the hypotheses of regularity of the power series method, in all above results the convergence in the sense of the power series method can be replaced by ordinary convergence as stated in the following theorem.

Theorem 5. Let $E, F$ be two Archimedean Riesz spaces, let $W$ be a nonempty subset of $E^{+}$, and let $\left(T_{n}\right)_{n}$ be a given sequence of elements of $\mathcal{L}(E, F)$ such that:

(a) $\left(T_{n}\right)_{n}$ converges pointwise on $W$ uniformly to $T \in \mathcal{L}(E, F)$.

(b) $T-T_{n}$ is an order bounded disjointness preserving operator.

(c) The power series method is regular.

Then $\left(T_{n}\right)_{n}$ converges pointwise on $E_{W}$ absolutely uniformly in the sense of the power series method to $T$. Thus,

$$
T x=\lim _{t \rightarrow R^{-}} \frac{1}{f(t)} \sum_{n=0}^{+\infty} a_{n} t^{n} T_{n} x
$$

for all $x \in E_{W}$.

As an application we give the following example which illustrates the importance of using the technique of convergence in the sense of the power series method. To the best of our knowledge, there is no proof without using this technique.

Example 6. Let $E$ be the Riesz space of all real-valued continuous functions $f$ on $[0,1]$ and assume that there exists a partition $\left\{x_{0}, \ldots, x_{n}\right\}$ of $[0,1]$ such that $f$ is linear on each interval $\left[x_{i-1}, x_{i}\right)$,i.e., $f(x)=m_{i}(f) x+b_{i}(f)$ for all $x \in\left[x_{i-1}, x_{i}\right)$. Now define for all $n \geq 1$

$$
\begin{aligned}
& T_{k}: E \rightarrow \mathbb{R} \\
& f \rightarrow f\left(\frac{k}{n+k}\right)
\end{aligned}
$$

and

$$
\begin{aligned}
& T: E \rightarrow \mathbb{R} \\
& f \rightarrow f(1)
\end{aligned}
$$

It is easily checked that $\left(T_{k}\right)_{k}, T \in \mathcal{L}(E, \mathbb{R}),\left(T_{k}\right)_{k}$ converges pointwise uniformly on $E$ to $T \in \mathcal{L}(E, \mathbb{R})$ and $T-T_{k}$ is an order bounded disjointness preserving operator for all $k \geq 1$. Now if $a_{k}=C_{n+k}^{n}$, then $f(t)=\sum_{k=0}^{+\infty} a_{k} t^{k}=$ $\frac{1}{(1-t)^{n+1}}$ for all $t \in(-1,1)$. It is easy to see that $\lim _{t \rightarrow 1^{-}} \frac{a_{k} t^{k}}{f(t)}=0$. Thus the power series method is regular, and hence Theorem 5 implies that

$$
f(1)=\lim _{t \rightarrow 1^{-}}(1-t)^{n+1} \sum_{k=0}^{+\infty} C_{n+k}^{n} f\left(\frac{k}{n+k}\right) t^{k}
$$

for all $f \in E$ and $n \geq 1$.

Acknowledgement: The authors wish to express their thanks to the referee for stimulating comments and suggestions on the first version of this paper.

\section{References}

[1] Korovkin B., On convergence of linear positive operators in the space of continuous functions, Doklady Akad. Nauk SSSR (N.S.), 1953, 90, 961-964

[2] Wolff M., On the universal Korovkin closure of subsets in vector lattices, J. Approx. Theory, 1978, 22, 243-253

[3] Altomare F., Korovkin-type and approximation by positive linear operators, Surv. Approx. Theory, 2010, 5, 92-164 
[4] Duman O., Orhan C., An abstract version of the Korovkin approximation theorem, Publ. Math. Debrecen, 2006, 69, 33-46

[5] Guessab A., Schmeisser G., Two Korovkin-type theorems in multivariate approximation, Banach J. Math. Anal., 2008, 2, 221-128

[6] Atlihan O. G., Tas E., An abstract version of the Korovkin theorem via A-summation process, Acta Math. Hungar., 2015, 145, 360-368

[7] Wisniewska H., Wojtowicz M., Approximations of the Korovkin type in Banach lattices, Rev. R. Acad. Cienc. Exactas Fis. Nat. Ser. A Mat. RACSAM, 2015, 109(1), 125-134

[8] Dorai A., Chil E., Wojtowicz M., Korovkin-type approximation theory in Riesz spaces, Mediterr. J. Math., 2018, 15:169

[9] Bardaro C., Boccuto A., Dimitriou X., Mantellini I., Abstract Korovkin-type theorems in modular spaces and applications, Cent. Eur. J. Math., 2013, 11(10), 1774-1784

[10] Ozguc I., Tas E., A Korovkin-type approximation theorem and power series method, Results Math., 2016, 69(3-4), 497-504

[11] Pinar O. S., Fadime D., A Korovkin-type theorem for double sequences of positive linear operators via power series method, Positivity, 2018, 22, 209-218

[12] Yurdakadim T., Abstract Korovkin theory in modular spaces in the sense of power series method, Hacet. J. Math. Stat., 2018, 47(6), 1467-1477

[13] Tas E., Yurdakadim T., Approximation by positive linear operators in modular spaces by power series method, Positivity, 2017, 21(4), 1293-1306

[14] Aliprantis C. D, Burkinshaw 0., Positive Operators, Academic Press, Orlando, 1985

[15] Luxemburg W. A., Zaanen A. C., Riesz Spaces I, North-Holland, Amsterdam, 1971

[16] Boos J., Classical and Modern Methods in Summability, Oxford Univ. Press. UK, 2000 